\title{
Photonic Molecules: A New Concept for Wavelength Tunable Optical Delay
}

\author{
John F. Donegan ${ }^{1}{ }^{1}$, Member IEEE, Yury P. Rakovich ${ }^{1}$, Matthias Gerlach ${ }^{1}$, Terry Connolly ${ }^{2}$ \\ John J. Boland ${ }^{2}$, Nikolai Gaponik ${ }^{3}$, and Andrey L. Rogach ${ }^{4}$ \\ ${ }^{1}$ Semiconductor Photonics Group, Department of Physics, Trinity College Dublin, Dublin 2, Ireland \\ ${ }^{2}$ Nanoscale Processing and Characterization Group, Department of Chemistry \\ Trinity College Dublin, Dublin 2, Ireland \\ ${ }^{3}$ Institute of Physical Chemistry, University of Hamburg, Grindelallee 117, 20146 Hamburg, Germany \\ ${ }^{4}$ Photonics \& Optoelectronics Group, Physic Department and CeNS, University of Munich \\ Amalienstr. 54, 80799 Munich, Germany \\ *Tel.+353-1-6081987, Fax. +353-1-6711759, email: jdonegan@tcd.ie
}

\begin{abstract}
The paper describes and light propagation modes in coherently coupled spherical microcavities covered with CdTe nanocrystals. We observe a rich new spectrum of resonances due to the lower symmetry of the two coupled microspheres: the photonic molecule. The quality factor $\mathrm{Q}$ of these new resonances are much greater than in the parent microspheres, indicating the possibility of using such coupled microcavities for the delaying of optical siganls. The large number of resonances shows the possibility to have wavelength selectivity in the photonic molecule structure. In addition, we show the potential for wavelength switching with the photonic molecule structure.
\end{abstract}

Keywords: integrated optics, microspheres, photonic molecules, nanocrystals, delay lines.

\section{INTRODUCTION}

At present, there is strong interest in research on the design and fabrication of optical structures for manipulation of light on micrometer-length scale. Much of this attention has been focused on photonic band-gap structures or two-dimensional photonic wires due to the possibility of their use in optical interconnection [1]. A closely related area is the research and development on the modification of photon modes in spherical microcavities, both for studies of fundamental optical properties and for potential applications [2]. It has been shown that the luminescence from semiconductor nanocrystals (NC) such as CdSe or CdTe, can be modified when these nanoemitters are placed on the surface of spherical microcavities which support emission in discrete optical modes known as whispering gallery modes (WGM) [3]. Following the analogy with quantum mechanics, we have three integers, $n, l$ and $m$, describing these WGM. These integers correspond to angular, radial and the azimuthal quantum numbers, respectively. The possibility for coherent coupling between WGM of two adjacent spherical particles forming "photonic molecule (PM)" has been investigated recently [4,5]. Theoretical [6] and experimental studies [5,7] show a rich spectrum of photonic states in the PM. In this paper we report on a detailed analysis of the $m$-resonances in PL spectra of PM, formed from two spherical microcavities with CdTe NCs and investigate the use of the PM for optical delay. In addition, we show how to use the PM for space selective wavelength switching.

\section{EXPERIMENTAL DETAILS}

For our studies, aqueous dispersions of melamine-formaldehyde (MF) microspheres, of $3 \pm 0.05 \mu \mathrm{m}$ in diameter, were combined with luminescent CdTe NCs using a layer-by-layer deposition technique [5, 7]. A colloidal solution of CdTe NCs, with a PL maximum at $563 \mathrm{~nm}(2.9 \mathrm{~nm}$ radius $)$ and a PL quantum efficiency of $\sim 15 \%$ at room temperature, was used for coating MF microspheres. The PL spectra from the microspheres and PM were recorded using a RENISHAW micro-PL system equipped with a positioning stage and an $\operatorname{Ar}^{+}$laser $(\lambda=488 \mathrm{~nm}$, $1.0 \mathrm{~mW}$ power).

\section{RESULTS AND DISCUSSION}

To investigate the photon modes in interacting spherical microcavities, we have measured PL spectra scanning a sample along the longitudinal axis of the PM Fig. 1. One can clearly see a number of narrow peaks arising from TE and TM WGM of the individual spheres. The pronounced double structure, with the intensity distribution dependent on the excitation position Fig. 2, is just a result of superposition of the uncoupled WGMs of individual microspheres. For a given excitation and detection configuration, we did not observe any new peaks in the PL spectra of the PM as compared with that of the single spheres which is an indication of weak intermode coupling. The shift in position of the $T E_{22}^{1}, T E_{23}^{1}, T M_{21}^{1}$ and $T M_{22}^{1}$ modes of single microspheres, 
which can be clearly seen in Fig. 2 is a result of a difference in the size of the two microspheres. Using Mie theory we find that the difference in size is only $11 \mathrm{~nm}$.

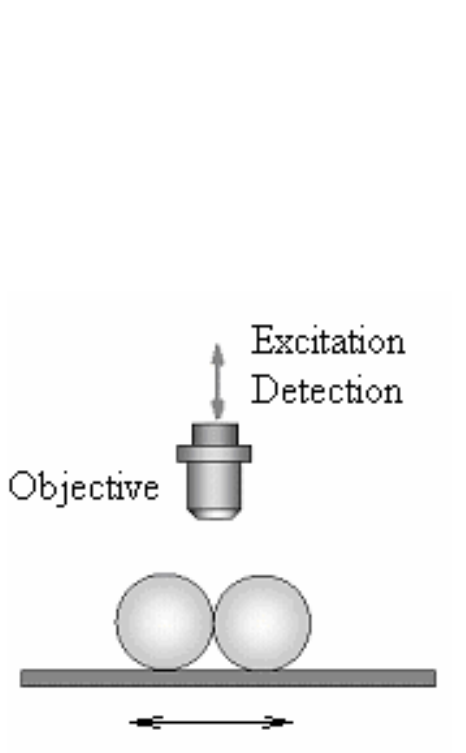

Figure 1. Experimental geometry for scanning PM along the longitudinal axis.

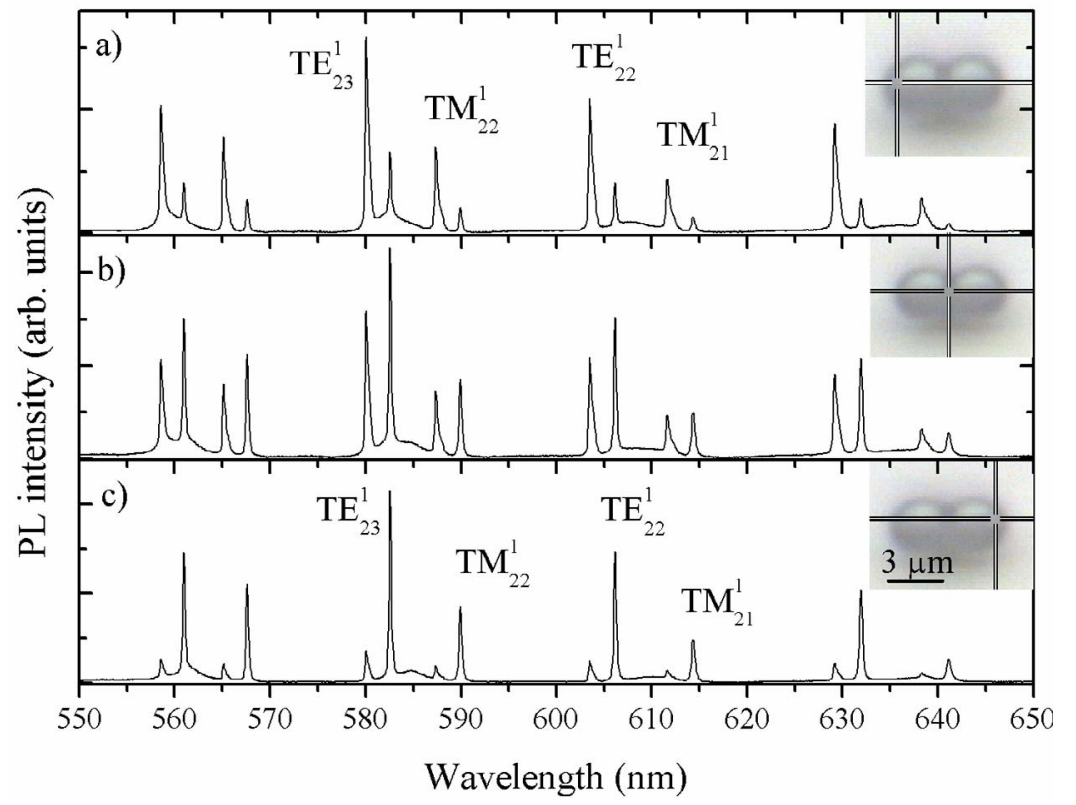

Figure 2. PL spectra of a PM with excitation and detection at three different positions along its longitudinal axis. Insets: microscope images of the PM, with the cross-hairs indicating the excitation-detection position.

As predicted in Ref. [6], a controllable alignment geometry of the PM is crucial in order to observe the strong coupling between the spheres. The signal from the coupled modes is expected to be more pronounced when the incident light propagates parallel to the longitudinal axis of the PM. In order to control the alignment of the spheres constituting the PM we utilized a polystyrene substrate containing a three-dimensionally ordered array of pores of $\sim 5 \mu \mathrm{m}$ in size prepared through a thermocapillary convection $[5,7]$. The surface layer of the substrate contains an array of open micro-wells of $3-5 \mu \mathrm{m}$ depth. Only one pair of the $3 \mu \mathrm{m}$ microspheres can be accommodated within each microwell, and the axis of the PM is close to the surface normal (Fig. 3a), inset).

Figure 3a shows the PL spectrum of a PM accommodated in a microwell and the spectra of the individual microspheres prior to being manipulated into the microwell. The presented PL spectra clearly reveal the major features unique to strong coherent coupling between the photonic states of the two microspheres forming the PM. One can clearly see a number of very narrow peaks ( $m$-resonances) in the spectrum of the PM on the short wavelength side of the TE and TM resonances, which are due to the presence of $m \neq \pm 1$ components. These peaks are grouped into the bonding $\mathrm{BN}$ and anti-bonding $\mathrm{ABN}$ branches following the analogy with molecular electronic structure (Fig. 3b).

For a single microsphere, the Mie resonance of a mode with angular quantum number $n$ has $(2 n+1)$-fold degeneracy and each degenerate state can be labeled by $m$ with $2 n<m<n$. The strong interaction between two microspheres changes the symmetry of the system and breaks the degeneracy of photonic states with respect to the $m$-index. Due to the dependence of the mode coupling on the orbital plane orientation, the degeneracy of PM modes is now $n+1$ with $m$ running from 0 to $n$ [6]. Now the interaction is limited to modes of the same $m$ and no degeneracy is removed between $m$ and $-m$. The variety of $m$-dependent internal field patterns causes a corresponding multi peak structure in PL spectra of the PM, which is very different from the WGM structure of individual microspheres. 


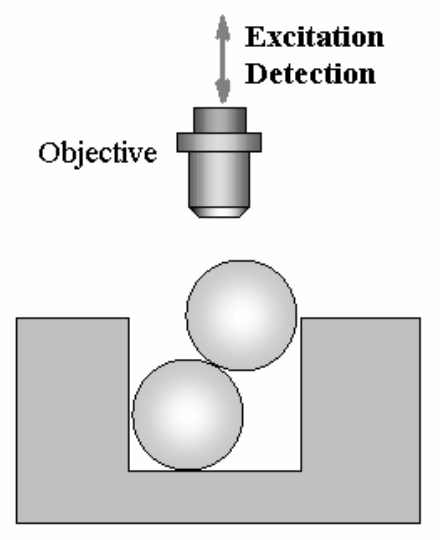

Figure 3a. Experimental geometry for off-axis excitation and detection geometry.

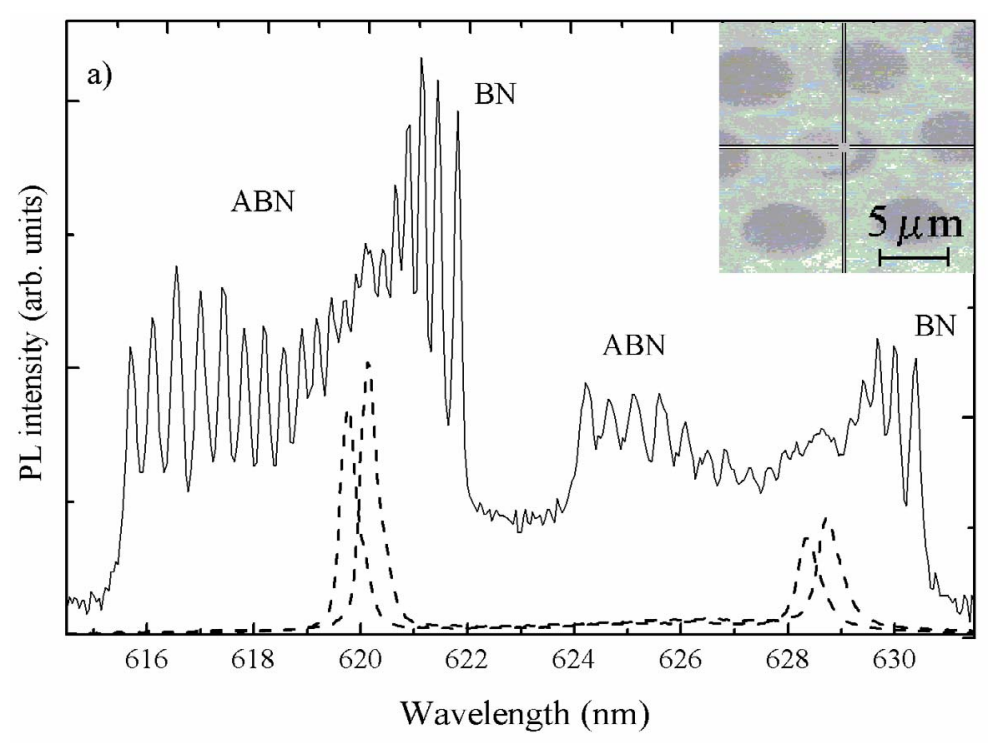

Figure 3b. Solid line: PL spectra of a PM accommodated in a microwell (inset). Dashed lines: spectra of non-interacting microspheres.

The deconvolution of the lineshape of the $m$-resonances of the PM using Lorentian functions reveals a remarkable experimental fact: the Q factor of $m$-resonances in the spectra of the PM $(\sim 8000)$ (and therefore the cavity lifetime, $\tau$, of a photon in the resonant modes) exceeds the $Q$ value $(\sim 3000)$ and photon lifetime of individual spheres across the whole spectral region. This fact along with estimated value of BN/ABN splitting $(\sim 6-8 \mathrm{~nm})$ suggests the possibility for the development of new PM-based photonic devices such as an optical delay line with controllable spectral and temporal tunability. The delay can be controlled using the sphere size, sphere separation and the angle of the PM with respect to the excitation.

In our experiments we have investigated the strong coupling phenomenon studying the spectra of PM in the visible region. In order to study the feasibility of using a PM as a optical delay device we have theoretically analysed the $m$-dependent structure for modes lying within the $\mathrm{C}$ band (approximated from $1525-1565 \mathrm{~nm}$ ): the low attenuation window which is traditionally used for optical communication.

Using the single-mode tight-binding method [6] we have calculated the spacing between adjacent $m$-modes (Fig. 4a) and the corresponding distribution of delay times (Fig. 4b). For simplicity in our simulations, we have considered only the case of the identical spheres with WGM having radial quantum number $\ell=1$, taking into account only the interaction between WGM of the same $n$. It can be noted from Fig. 4a and Fig. 4b that the nonuniform spacing between $m$-resonances of the PM is accompanied by a non-uniform delay time distribution. For the smallest PM size, 18 channels are available within a "window" of $12-200 \mathrm{GHz}$. The smaller Q-factor of the WGM in these spheres results in relatively modest increase in delay time - it grows from 1.5 ps (obtained for ABN resonance with $m=1$ ) up to $4.7 \mathrm{ps}$ calculated for corresponding $m$-resonance of BN mode of PM. For microspheres of larger size, the number of channels available increases. Indeed, for 12- $\mu \mathrm{m}$ coupled spheres, 20 channels fit into above indicated spacing window, with biggest value of channel spacing $\sim 130 \mathrm{GHz}$. The delay times distributed between these channels grow from 5.2 ps up to 16.3 ps. The upper limit of channel spacing drops further for a PM formed from 16- $\mu \mathrm{m}$ microspheres. In that case, 22 channels with spacing between 12 and $76 \mathrm{GHz}$ can be seen in Fig. 4a, providing the same number of discrete time delays which were found to be distributed between 94.5 ps and 297 ps. The maximum delay provided by this PM corresponds to a $\sim 6 \mathrm{~cm}$ length of silica-glass waveguide delay line.

Returning now to Fig. 2, we find that when the excitation is provided at the contact point between microspheres (Fig. 2b), the excited and emitted light propagate efficiently along the longitudinal axis of the PM and the contribution of both microcavities can be clearly seen in the PL spectra at discrete WGM wavelengths. This contribution to the PL signal from individual microcavities can be switched by changing excitation position from the left to the right rim of the PM (Fig. 2a, Fig. 2c). The lack of intermode coupling in this configuration is not surprising because the coupling between electro-magnetic fields of the spheres is expected to be maximum in the direction parallel to the PM axis, when some of WGM orbitals lie in the same plane [6]. However, the observed redistribution of intensity between the components of the WGM double structure clearly demonstrates the waveguiding of the light along the PM and the possibility of wavelength switching in the PM depending on excitation-detection geometry. 


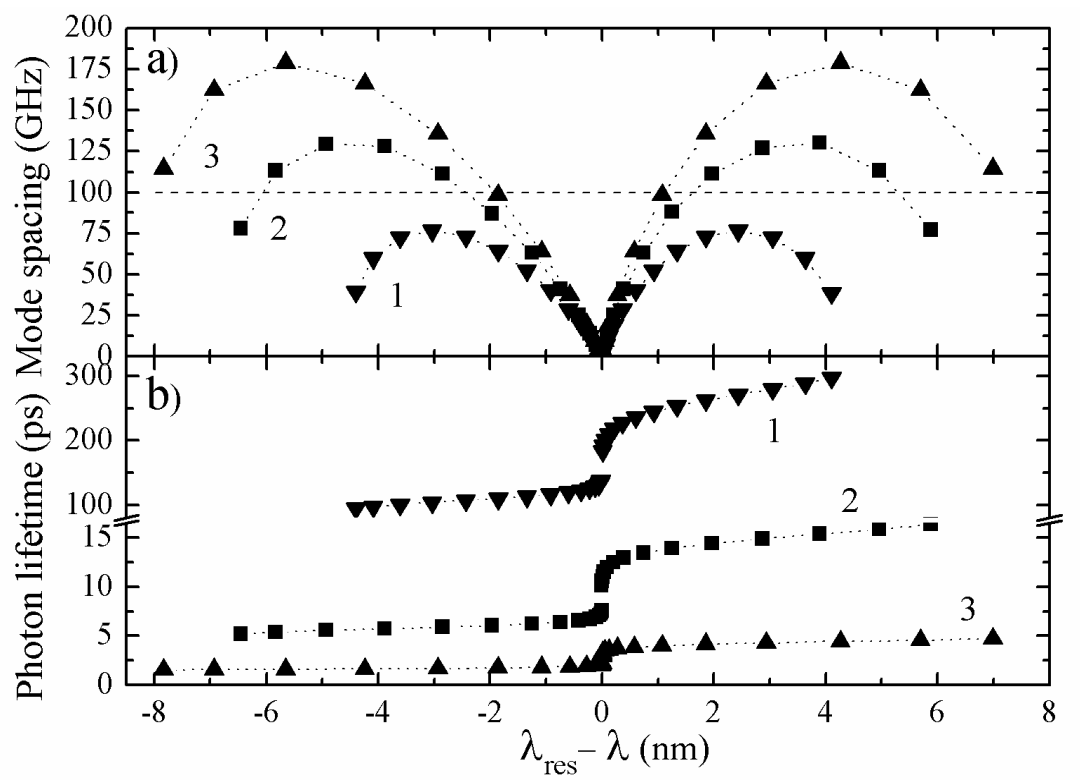

Figure 4. Spacing between adjacent m-modes of PM formed by two identical microspheres with sizes of 10 (1), 12 (2) and 16 (3) $\mu \mathrm{m}$ (a) and calculated distribution of delay time (b). The zero value on wavelength axis corresponds to the position of $T E_{24}, T E_{29}$ and $T E_{40} W G M$ of the single spherical microcavity.

\section{CONCLUSIONS}

Using these results we can draw a few general conclusions. Two coherently coupled spherical microcavities can generate modal structure with quality factors and photon lifetimes significantly higher than that of single microspheres. By analysing the $m$-dependent splitting of the $\mathrm{BN}$ and $\mathrm{ABN}$ modes of the PM with size comparable to core of an optical fiber, we have introduced a new strategy for potential application of coupled spherical microcavities as a multi-channel, wavelength-tunable optical delay device, which does not require an ultra-high Q-factor for the microspheres. In the proposed scheme, the bandpass width, channel spacings and delays depend only on the physical parameters of the interacting microcavities.

\section{ACKNOWLEDGEMENT}

This work was supported by Science Foundation Ireland under grant numbers 02/IN.1/I47 and 00/PI.1/C077A.2 and by the Deutsche Forschungsgemeinschaft through the SPP "Photonic Crystals".

\section{REFERENCES}

[1] J.D. Joannopoulos, P.R. Villeneuve, S. Fan 'Photonic crystals: putting a new twist on light', Nature, vol. $386,143-149,1997$

[2] K.J. Vahala 'Optical microcavities', Nature, vol. 424, 839-846, 2003.

[3] M. Artemyev, U .Woggon 'Quantum dots in photonic dots', Appl. Phys. Lett., vol.76, 1353-1355, 2000.

[4] M. Bayer, T. Gutbrod, J.P. Reithmaier, A. Forchel, T.L. Reinecke, P.A. Knipp, A.A. Dremin, V.D. Kulakovskii 'Optical modes in photonic molecules', Phys. Rev. Lett., vol. 81, 2582-2585, 1998.

[5] Y.P. Rakovich, M. Gerlach, A.L. Bradley, J.F. Donegan, T.M. Connolly, J.J. Boland, M.A. Przyjalgowski, A. Ryder, N. Gaponik, A.L. Rogach 'Confined optical modes in small photonic molecules with semiconductor nanocrystals', J. Appl. Phys., vol. 96, 6761-6765, 2004.

[6] H. Miyazaki, Y. Jimba 'Ab initio tight-binding description of morphology-dependent resonance in a bisphere', Phys. Rev. B., vol. 62, 7976-79976, 2000.

[7] Y.P. Rakovich, J.F. Donegan, M. Gerlach, A.L. Bradley, T.M. Connolly, J.J. Boland, N. Gaponik, A. Rogach 'Fine structure of coupled optical modes in photonic molecules', Phys. Rev. A, vol. 70, 051801-1/4, 2004. 